\title{
Prostate specific antigen and acinar density: a new dimension, the "Prostatocrit"
}

\author{
Simon Robinson ${ }^{1}$, Marc Laniado ${ }^{1}$, Bruce Montgomery ${ }^{1}$ \\ ${ }^{1}$ Frimley Park Foundation Trust, United Kingdom
}

\section{ABSTRACT}

Background: Prostate-specific antigen densities have limited success in diagnosing prostate cancer. We emphasise the importance of the peripheral zone when considered with its cellular constituents, the "prostatocrit".

Objective: Using zonal volumes and asymmetry of glandular acini, we generate a peripheral zone acinar volume and density. With the ratio to the whole gland, we can better predict high grade and all grade cancer. We can model the gland into its acinar and stromal elements. This new "prostatocrit" model could offer more accurate nomograms for biopsy.

Materials and Methods: 674 patients underwent TRUS and biopsy. Whole gland and zonal volumes were recorded. We compared ratio and acinar volumes when added to a "clinic" model using traditional PSA density. Univariate logistic regression was used to find significant predictors for all and high grade cancer. Backwards multiple logistic regression was used to generate ROC curves comparing the new model to conventional density and PSA alone.

Outcome and results: Prediction of all grades of prostate cancer: significant variables revealed four significant "prostatocrit" parameters: log peripheral zone acinar density; peripheral zone acinar volume/whole gland acinar volume; peripheral zone acinar density/whole gland volume; peripheral zone acinar density. Acinar model (AUC 0.774), clinic model (AUC 0.745) $(\mathrm{P}=0.0105)$.

Prediction of high grade prostate cancer: peripheral zone acinar density ("prostatocrit") was the only significant density predictor. Acinar model (AUC 0.811), clinic model (AUC 0.769) $(\mathrm{P}=0.0005)$.

Conclusion: There is renewed use for ratio and "prostatocrit" density of the peripheral zone in predicting cancer. This outperforms all traditional density measurements.

\section{ARTICLE INFO}

Keywords:

AcinarCells; Prostatic Neoplasms; Diagnosis; PSA

Int Braz J Urol. 2017; 43: 230-8

\section{Submitted for publication:}

April 12, 2016

Accepted after revision:

October 15, 2016

Published as Ahead of Print: January 05, 2017

\section{INTRODUCTION}

PSA and derived densities, whole gland density, PSAD $(1,2)$ and the transition zone density PSATD (3), have a limited role in diagnosing cancer despite initial optimism. This is partly due to age related changes (4).We propose a new way of using the zones of the prostate taking into account their absolute volumes and the asymmetry in the amount of glandular acini within each (5-7). Hence the relative contribution of each zone, in terms of both epithelial acinar cells and their PSA production, to the entire gland. This highlights the contribution of the peripheral zone acinar volume (PZav) and its acinar density (PZad). We divide the serum 
PSA into the differing amounts of acini within zones of differing volumes.

The peripheral zone is an intrinsically more stable entity (in terms of volumes and relative amounts of stroma and acini). This allows an intuitive approach to use volumes with appropriate densities (not the arbitrary divising of entire PSA into the transition zone). It is these acinal cells that produce PSA, a marker of epithelial activity, and which undergo mitotic events causing cancer. Regardless of the nature of the nodule, there is always an increase in stroma relative to epithelium (8) and a diluting effect on glandular components. We have taken the overall glandular quantity in the entire gland to be 70\%, most of which is concentrated in the peripheral zone $(6,8-10)$. This varies with age. The peripheral zone is far less variable in acini and stroma, and we extrapolate our other values from this constant entity.

We can better predict cancer of all grades, and more importantly, better predict high grade cancer than conventional densities. Finally, we propose that we will be able to model both the benign gland as it ages, in terms of each zone, its divisions into acinar and stromal elements, and further, that we can contrast this with the relative growth dynamics of the malignant gland.

\section{MATERIALS AND METHODS}

Our study population included 672 patients admitted to a district general hospital, for transrectal ultrasound and biopsy, from 2007 to 2012, because of elevated PSA, anxiety or abnormal rectal exam. This was performed by one physician. The inner gland and the outer, peripheral zone were measured. 409 were benign and 263 malignant. We measured the whole gland volume (WGv) using the ellipsoid formula, then the inner gland (transition zone and central gland combined). We subtracted this from the WGv to yield the peripheral zone volume (PZv). We documented those with a positive family history, a first degree relative affected by prostate cancer. Also whether they had had a previous negative biopsy and whether the prostate felt suspicious of cancer or was clearly malignant (Table-1).

We have accepted the established glandular, acinar component of the prostate of 70\%. The WGv x 0.7 yields the whole gland acinar volume (WGav). By definition, 1-WGav yields the whole gland stromal volume (WGsv). The peripheral zone was attributed a percentage of $80 \% .0 .8 \mathrm{x}$ $\mathrm{PZv}$ yields the peripheral zone acinar volume (PZav).

1-PZav yields the peripheral zone stromal volume (PZsv). The WGav - PZav yields the transition zone acinar volume (TZav).

1-TZav yields the transition zone stromal volume (TZsv) (Figure-1). Figure-2 demonstrates how the zones vary with a growing gland.

\section{Densities}

The serum PSA is divided into WGav to yield the whole gland acinar density. The peripheral zone acinar density is calculated by multiplying the WGad times the ratio of the PZav/WGav.

The transition zone acinar density is WGad-PZad.

Formula:

$$
\begin{aligned}
& 0.7 \mathrm{WGv}=\mathrm{WGav} \\
& \mathrm{PSA} / \mathrm{Wgav}=\mathrm{WGad} \\
& 0.8 \mathrm{PZv}=\mathrm{PZav} \\
& \text { Wgav }-\mathrm{Pzav}=\mathrm{TZav} \\
& \mathrm{WGad} \times \mathrm{PZav} / \mathrm{Wgav}=\mathrm{PZad} \\
& \mathrm{WGad}-\mathrm{Pzad}=\mathrm{TZad} \\
& \mathrm{WGv}-\mathrm{Wgav}=\mathrm{WGsv} \\
& \mathrm{TZv}-\mathrm{Tzav}=\mathrm{TZsv} \\
& \mathrm{PZv}-\mathrm{Pzav}=\mathrm{PZsv}
\end{aligned}
$$

\section{Statistics}

Univariate logistic regression was initially used to find significant predictors for all grades of prostate cancer and then for high grade (Gleason 7 and above). Backwards multiple logistic regression was used to generate ROC curves using the new model and comparing it to whole gland density, PSA density and to PSA alone using Medcalc statistical software. 
Table 1 - Patient characteristics.

\begin{tabular}{lccc}
\hline & & BPH & PC \\
\hline Age years & Mean & 63.0 & 68.1 \\
& Median & 63.0 & 68.0 \\
PSA ng/mL & Interquartile range & $58-68$ & $63-75$ \\
& Mean & 8.1 & 16.4 \\
& Median & 6.6 & 8.5 \\
Whole gland volume cc & Interquartile range & $4.6-9.1$ & $5.8-13.3$ \\
& Mean & 57.6 & 48.0 \\
Transition zone volume cc & Median & 51.0 & 40 \\
& Interquartile range & $35-71$ & $29-59$ \\
Peripheral zone volume cc & Mean & 29.8 & 22.4 \\
& Median & 24.0 & 16.0 \\
& Interquartile range & $14-39.5$ & $9-29$ \\
Previous negative biopsy & Mean & 27.7 & 25.7 \\
Family history & Median & 24.0 & 24.0 \\
DRE & Interquartile range & $18-34$ & $17-31$ \\
& & $93(23 \%)$ & $73(28 \%)$ \\
& & $48(12 \%)$ & $37(14 \%)$ \\
\hline
\end{tabular}

\section{RESULTS}

See Table 1

See Table 2

See Table 3

See Figure 3

See Figure 4

\section{DISCUSSION}

Developing the model

To tackle the problem of PSA and its variable zonal densities, we need to take into account the distribution of acini within the gland in the normal man and the age related changes of hyperplasia within nodules. The periphery was noted, in autopsies, to account for most of the glandular material (6) and the inner gland to account for most of the ducts and stroma. The asymmetry of gland distri- bution was further enforced when normal (autopsy) and enlarged (suprapubic prostatectomy) glands were compared (8). In the glands with benign prostatic hyperplasia, the glandular component was only $12 \%$ and this was mostly due to "dilution" by stromal growth. Growth rates were elucidated showing two phases, a rapid increase in growth from ten years old to thirty years then a slow gradual increase from thirty onwards. The normal stroma constituting about 50\% of the mass and epithelium and acinal lumen the other 50\%. Hyperplasia is associated with growth of both components to varying degrees (11). The ratio of stroma to epithelium, within adenomas, varies with symptoms (4.6) and without symptoms (2.6) (12). The stroma forms $62 \%$ of the symptomatic gland and epithelium 38\%. The conclusion is that $\mathrm{BPH}$ is primarily a stromal process. Using enzymatic staining it was found the stroma to constitute $76 \%(12,13)$ and that it is the ratio increase leading to symptoms. 
Figure 1 - Normal gland 20cc schematic diagram. 70\% PZ by volume $30 \%$ TZ by volume. PZ $80 \%$ acini $20 \%$ stroma (cells red, lumen black, stroma pink).

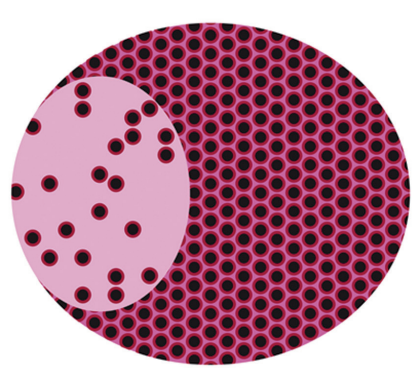

20cc whole gland peripheral zone $70 \%$

That PSA is related to prostate size, the amount of epithelium and testosterone was well established. But size is not a good predictor of PSA because of the great variation in acini and whole gland volume with a range of stromal component (14). It was proposed that epithelium itself is not a good guide to the amount of PSA because the architecture of acini can be disrupted by disease processes both benign and malignant. Further, PSA secretion is androgen independent and this wanes with advancing years.

The predominance of epithelium within the PZ compared to TZ, yet the lack of correlation of PSA with the PZ and the greater correlation of PSA with TZ has been confirmed (7). Further, the variability of the $\mathrm{TZ}$ and the relative constancy of the $\mathrm{PZ}$ is documented. The confounding problem of PSA is that it appears to be highly dependent on the $\mathrm{TZ}$ rather than the $\mathrm{PZ}$. The rate of $\mathrm{BPH}$ epithelium growth is $\mathrm{x} 9$ the normal gland rate and stromal growth within BPH x 37 the normal rate (15). In regard to ratio, the converse is true for cancers.

The androgen receptor is present in both stroma and epithelium, but 5 alpha reductase is only present in stromal cells and that they have an inductive influence on the epithelium (16). The

Figure 2 - Prostate gland with variation in whole gland size and peripheral zone as $20,40,60 \%$ of whole gland volume.

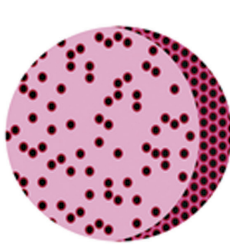

20cc whole gland peripheral zone $20 \%$



$40 \mathrm{cc}$ whole gland

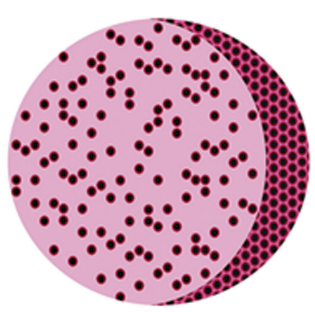
peripheral zone $20 \%$

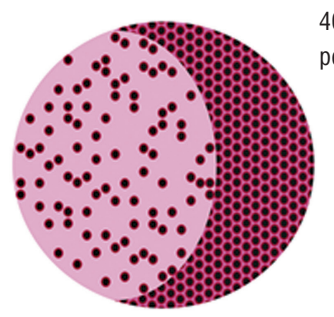

$40 \mathrm{cc}$ whole gland peripheral zone $40 \%$

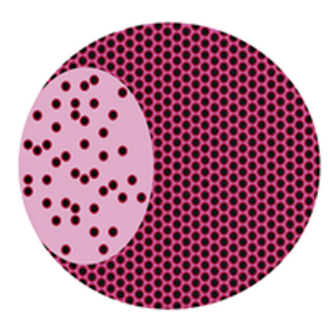

$40 \mathrm{cc}$ whole gland peripheral zone $60 \%$


Whole gland volume 20cc top row; Whole gland volume 40cc middle row; Whole gland volume 80cc bottom row 
Table 2 - All grade and high grade models.

\begin{tabular}{lcccccc}
\hline $\begin{array}{l}\text { All grade } \\
\text { model }\end{array}$ & Variable & Coefficient & Standard error & $P$ & Odds ratio & $\begin{array}{c}\text { Confidence } \\
\text { interval }\end{array}$ \\
\hline \multirow{5}{*}{} & Age & 0.089 & 0.01 & $<0.0001$ & 1.09 & $1.067-1.11$ \\
& DRE = cancer & 2.14 & 1.07 & 0.0466 & 8.5 & $1.03-70.0$ \\
& Family history & 0.57 & 0.27 & 0.0382 & 1.7 & $1.03-3.08$ \\
& positive & & & & & \\
& LnPZAd & 0.58 & 0.16 & 0.0003 & 1.8 & $1.30-2.48$ \\
& Previous & -0.55 & 0.24 & 0.0218 & 0.57 & $0.35-0.92$ \\
& negative biopsy & & & & & \\
& PZAv/WGAv & 1.43 & 0.62 & 0.0223 & 4.18 & $1.22-14.28$ \\
& PZAd/WGv & 60.1 & 26.23 & 0.0219 & $129 \mathrm{E}+24$ & $6058-2.77 \mathrm{E}+048$ \\
High grade & PZAd & -1.34 & 0.67 & 0.0470 & 0.26 & $0.06-0.98$ \\
model & Age & 0.06 & 0.014 & $<0.0001$ & 1.07 & $1.04-1.10$ \\
& DRE suspicious & 0.61 & 0.240 & 0.0103 & 1.85 & $1.15-2.96$ \\
& DRE cancer & 3.49 & 1.105 & 0.0015 & 33.08 & $3.79-288.7$ \\
& Ln PZad & 0.98 & 0.134 & $<0.0001$ & 2.67 & $2.05-3.49$ \\
& Previous & -0.69 & 0.330 & 0.0346 & 0.49 & $0.26-0.95$ \\
& negative biopsy & & & & & \\
\hline
\end{tabular}

Variables not included in all grade model; DRE suspicious; PSA; Race Caucasian; Race Afro-Caribbean; PZD; TZD; PSAD (WGD)

Variables not included in high grade model; family history; PSA; PZAD/WGV; PZAV/WGAV; PZD; Race Caucasian; Race Afro-Caribbean; TZD; PSAD (WGD)

Table 3 - Positive and negative predictive values (PZad).

\begin{tabular}{lccccccccc}
\hline \multicolumn{4}{c}{ High grade prostate cancer } & \multicolumn{4}{c}{ All grades of prostate cancer } \\
\hline Mean PZad & $\begin{array}{c}\text { No } \\
\text { cancer }\end{array}$ & Cancer & Total & PPV\% & NPV\% & No cancer & Cancer & PPV\% & NPV\% \\
\hline 0.043 & 128 & 7 & 135 & 5 & 95 & 104 & 31 & 23 & 77 \\
0.082 & 118 & 17 & 135 & 13 & 87 & 101 & 34 & 25 & 75 \\
0.121 & 115 & 19 & 134 & 14 & 86 & 92 & 42 & 31 & 69 \\
0.197 & 106 & 29 & 135 & 21 & 79 & 73 & 62 & 46 & 54 \\
0.775 & 74 & 61 & 135 & 45 & 55 & 40 & 95 & 70 & 30 \\
\hline
\end{tabular}

range of influencing factors are categorised as intrinsic and extrinsic and there is no simple relation to androgen levels (17). We see in our cohort that the BPH glands are bigger overall, with larger transition zones, a similar size of peripheral zone and that the patients are younger than those with malignant glands (Table-1).

Ever since PSA was first localized to the gland (18) its exact use in diagnosis has been hampered by a lack of cancer specificity (1).
The relation of serum PSA to the entire gland volume was proposed (2) to allow an individualised approach to patients with intermediate PSA levels. The relation of PSA to zones of the gland was established using correlation coefficients (4). When reviewing patients who had undergone radical prostatectomy and cystectomy, it is apparent that the peripheral zone is significantly larger than the transition zone. It was found the average ratio of $\mathrm{TZ}$ to $\mathrm{PZ}$ was 3:1. Part of the 
Figure 3 - ROC for all grades (AG) of cancer. Comparing acinar ratio (Prostatocrit) model with clinic model and PSA alone

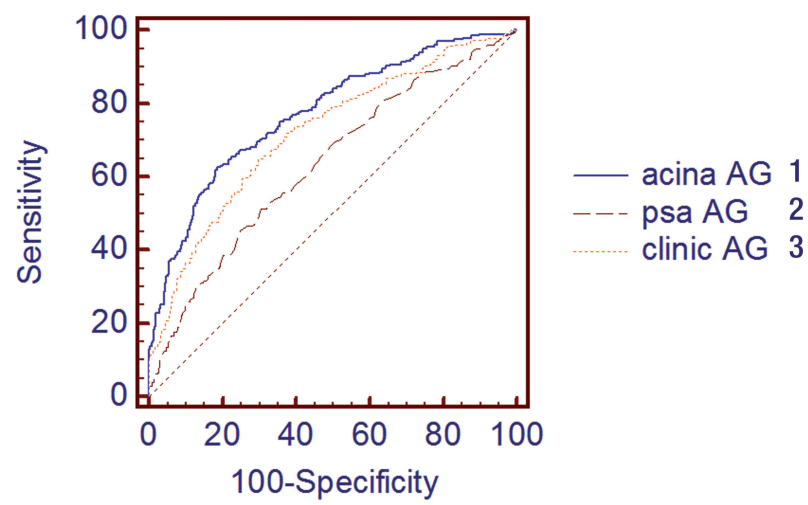

1 - Acinar prostatocrit model AUC 0.7742; 2 - PSA AUC $0.636 \mathrm{P}=0.0001 ; 3$ - Clinic model (PSAD) AUC $0.745 \mathrm{P}=0.01053$

Figure 4 - ROC for high grade cancer. Comparing prostatocrit model with clinic model and PSA alone.

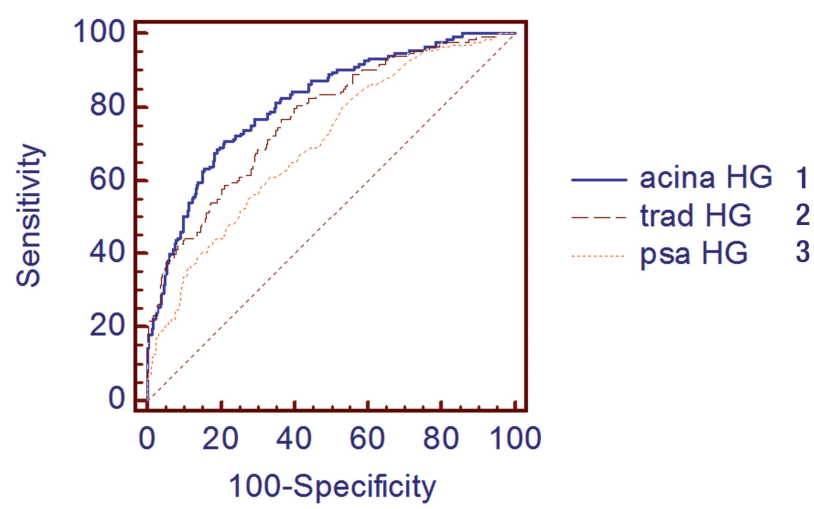

1 - Acina prostatocrit model AUC 0.811; 2 - Clinic (PSAD) model AUC $0.782 \mathrm{P}=$ $0.005 ; 3$ - PSA model AUC $0.700 \mathrm{P}=0.0001$

problem with assessing the gland is the great variability in the $\mathrm{TZ}$ ranging from $2-80 \%$ of the total volume (19, 20). Recent studies (21) fail to differentiate cancer using PSAD. Further refinement was attempted with free/total ratio and TZAD (22) with PSA in the range of $2.5-4 \mathrm{ng} / \mathrm{mL}$. However, this has great limitations with small glands. The relation of the whole volume to PSAD and TZAD was proposed (23) and improves specificity and can limit unnecessary biopsies. The role of the PSATD has been recently strengthened by showing it had the most predictive power in diag- nosing cancer (24). However, its use in predicting stage (25) reveals the confounding influence of the TZ. Regarding the PZ, it is suggested that the cancer which arises here, does so because of more cells of epithelial origin that are undergoing cell division and potential cancerous changes will be more numerous here. It appears there is a difference in the ratio of the two zones in cancer patients compared to benign patients. The peripheral zone is intrinsically richer in the acini that make PSA (5). The whole issue of PSA density and zonal densities has been dominated by the adoption of the term transition zone density which divides the entire serum PSA into the volume occupied by the inner gland $(3,26,27)$. This is an incorrect use mathematically. Practically, it can contribute to diagnosis $(26,7)$, but ideally, the relative contribution of the separate zones should be accounted for. This is intuitively confusing otherwise and we end up with total densities greater than the original. The division of total serum PSA into the TZ ignores the contribution of the PZ to serum PSA. The corresponding lack of use of the TZAD highlights this (28). The peripheral zone has had limited application so far, although it has proved useful in men on alpha reductase inhibitors (29).

To illustrate the problem, we compared the two approaches below for a 40cc gland with equal components of peripheral and transition zones and with a serum PSA of $4 \mathrm{ng} / \mathrm{mL}$.

Thus, $\mathrm{WGd}=0.1 \mathrm{ng} / \mathrm{mL} / \mathrm{cc}$

Traditional method

- $\mathrm{TZ}=20 \mathrm{cc} \mathrm{TZD}=0.2 \mathrm{ng} / \mathrm{mL} / \mathrm{cc}$

- $\mathrm{PZ}=20 \mathrm{cc} \mathrm{PZD}=0.2 \mathrm{ng} / \mathrm{mL} / \mathrm{cc}$

- Total density is now $0.4 \mathrm{ng} / \mathrm{mL} / \mathrm{cc}$

Prostatocrit method

- We have to take into account for the relative contributions of each zone

- The TD is $0.1 \times 20 / 40=0.05 \mathrm{ng} / \mathrm{mL} / \mathrm{cc}$

- The PD is $0.1 \times 20 / 40=0.05 n g / m L / c c$ total $=0.1 \mathrm{ng} / \mathrm{mL} / \mathrm{cc}$

We now have a density attributable to the zonal volume. This can be refined estimating the bulk of epithelium/acini within each zone.

We are aware of packed cell volume in haematology. The acini are equivalent to red cells, 
the stroma is equivalent to plasma and the PSA is equivalent to haemoglobin.

The gland is composed, overall, of 50-70\% acini. Having accounted for different zonal volumes, we now need to account for the asymmetry of distribution of acini (Figures 1 and 2).

The peripheral zone is denser in acini by definition. Let us assume it is $80 \%$ acini. The transition zone must be less dense. For demonstration purposes, we choose 60\% acini. The peripheral zone does not vary in its composition unlike the transition zone.

- For our 40cc gland with equal 20cc zones

- $\mathrm{TZ}=20 \mathrm{cc} \times 0.6=12 \mathrm{cc}$ acinar volume

- $\mathrm{PZ}=20 \mathrm{cc} \times 0.8=16 \mathrm{cc}$ acinar volume

- $\quad=28 \mathrm{cc}$ total acinar volume

This is the amount of epithelial tissue within the whole gland.

The density is derived by dividing the serum PSA into the relevant amount of acini.

- $\mathrm{WGad}=4 / 28=0.14 \mathrm{ng} / \mathrm{mL} / \mathrm{cc}$ of acini

- $\mathrm{TZad}=0.14 \times 12 / 28=0.06 \mathrm{ng} / \mathrm{mL} / \mathrm{cc}$

- $\mathrm{PZad}=0.14 \times 16 / 28=0.08 \mathrm{ng} / \mathrm{mL} / \mathrm{cc}$

Given that we can estimate the volume of acini within each zone, we can then by simple subtraction, estimate the amount of stroma, that is 1 -acina $\%$.

- $\mathrm{TZsv}=20-12=8 \mathrm{cc}$

- $\mathrm{PZsv}=20-16=4 \mathrm{cc}$

- $8+4=12$ cc stroma

- 12 cc stroma + 28cc acini=40cc whole gland.

\section{Using the model}

Univariate logistic regression for prediction of all grades of prostate cancer were used to test significant predictors in a multivariate logistic regression. There were four significant zonal predictors. All involved the peripheral zone. The log of the peripheral zone acinar density, the ratio of the peripheral zone acina volume to the whole gland acinar volume, the peripheral zone acinal density to the whole gland volume and the peripheral zone acinar density. None of the conven- tional zone densities, whole gland, transition or peripheral zone densities were included.

We compared ROC for this model with a "clinic" model using the same information: PSA, age, family history, previous negative biopsy, rectal examination and overall gland volume (Table- 2 and Figure-3). We also compared PSA on its own. There was a significant improvement in the area under the curve from PSA alone, to the conventional clinic model, to the prostatocrit model using ratio and acinar density of the PZ in four different combinations, all significant and all superior to traditional density measurements.

Most importantly, of practical clinical significance, it differentiated high grade (Gleason 7 and above) cancers better than traditional parameters. The only significant predictor was the peripheral zone acina density. None of the traditional densities were significant (whole gland, transition and peripheral zone densities) (Table-2 and Figure-4). The influence of the peripheral zone acinar density is further illustrated in Table-3, which shows the increasing positive predictive value as this density increases and the converse, the decreasing negative predictive value.

\section{Limitations}

TRUS is probably less accurate than MRI for measuring these volumes. These models will be improved with MRI and manual contouring of zones. We plan to do this in our next study. We also appreciate that other markers such as PCA3 and $4 \mathrm{~K}$ could be included. The correlation of predicted acina density and final actual histological density will potentially strengthen this model.

Assumption of equal production of PSA in all types of acini in both periphery and transition zone. The formula is complex and will be part of a calculator, but this should not concern the physician.

\section{Strengths}

This is an intuitive use of density and accounts for the relative constant amount of peripheral zone epithelium within an easily measured zone. The peripheral acinar zone was consistently significant in predicting all grade cancer and high grade cancer. It revealed four significant parameters all superior to traditional density measurements. 


\section{CONCLUSIONS}

When comparing the benign and malignant gland, the differences in ratio, with their acinal asymmetry, concentrated in the peripheral zone, enable the prostatocrit model to discriminate better between the two and hence who should have biopsies.

The absolute relation between zones, their acinal bulk and PSA production remains to be determined and may prove impractical, but this recognition of acinar contribution, may improve modelling of benign and cancerous disease, the response to drugs and need for surgery.

\section{Take home message}

PSA, density, zones, acinal asymmetry provides a new dimension to the analysis of the prostate gland. This prostatocrit model better predicts high grade cancer, all grades of cancer and it will help describe natural benign growth of the separate zones.

\section{CONFLICT OF INTEREST}

None declared.

\section{REFERENCES}

1. Stamey TA, Yang N, Hay AR, McNeal JE, Freiha FS, Redwine E. Prostate-specific antigen as a serum marker for adenocarcinoma of the prostate. $N$ Engl $J$ Med. 1987;317:909-16.

2. Benson MC, Whang IS, Olsson CA, McMahon DJ, Cooner WH. The use of prostate specific antigen density to enhance the predictive value of intermediate levels of serum prostate specific antigen. J Urol. 1992;147:817-21.

3. Kalish J, Cooner WH, Graham SD Jr. Serum PSA adjusted for volume of transition zone (PSAT) is more accurate than PSA adjusted for total gland volume (PSAD) in detecting adenocarcinoma of the prostate. Urology. 1994;43:601-6.

4. Hammerer PG, McNeal JE, Stamey TA. Correlation between serum prostate specific antigen levels and the volume of the individual glandular zones of the human prostate. J Urol. 1995;153:111-4.

5. Marks LS, Treiger B, Dorey FJ, Fu YS, deKernion JB. Morphometry of the prostate: I. Distribution of tissue components in hyperplastic glands. Urology. 1994;44:486-92.
6. McNeal JE. Origin and evolution of benign prostatic enlargement. Invest Urol. 1978;15:340-5.

7. Lepor H, Wang B, Shapiro E. Relationship between prostatic epithelial volume and serum prostate-specific antigen levels. Urology. 1994;44:199-205.

8. Bartsch G, Müller HR, Oberholzer M, Rohr HP. Light microscopic stereological analysis of the normal human prostate and of benign prostatic hyperplasia. J Urol. 1979;122:487-91.

9. Shapiro E, Becich MJ, Hartanto V, Lepor H. The relative proportion of stromal and epithelial hyperplasia is related to the development of symptomatic benign prostate hyperplasia. J Urol. 1992;147:1293-7.

10. Peterson R0, Sesterhenn IA, Davis CJ. Urologic Pathology.; 2009.

11. Berry SJ, Coffey DS, Walsh PC, Ewing LL. The development of human benign prostatic hyperplasia with age. J Urol. 1984;132:474-9.

12. Shapiro E, Hartanto V, Lepor H. Quantifying the smooth muscle content of the prostate using doubleimmunoenzymatic staining and color assisted image analysis. J Urol. 1992;147:1167-70.

13. Shapiro E, Hartanto V, Lepor H. Anti-desmin vs. anti-actin for quantifying the area density of prostate smooth muscle. Prostate. 1992;20:259-67.

14. Weber JP, Oesterling JE, Peters CA, Partin AW, Chan DW, Walsh PC. The influence of reversible androgen deprivation on serum prostate-specific antigen levels in men with benign prostatic hyperplasia. J Urol. 1989;141:987-92.

15. Issa. Contempory Diagnosis and Management of Diseases of the Prostate Anatomy of the Genitourinary System. 3rd ed. (Newtown PA Handbooks in healthcare co, ed.).; 2005.

16. Lee C, Kozlowski JM, Grayhack JT. Etiology of benign prostatic hyperplasia. Urol Clin North Am. 1995;22:237-46.

17. Lee C, Kozlowski JM, Grayhack JT. Intrinsic and extrinsic factors controlling benign prostatic growth. Prostate. 1997;31:131-8.

18. Ford TF, Butcher DN, Masters JR, Parkinson MC. Immunocytochemical localisation of prostate-specific antigen: specificity and application to clinical practice. $\mathrm{Br} \mathrm{J}$ Urol. 1985;57:50-5.

19. Zlotta AR, Schulman CC. [Use of transrectal ultrasonography in prostate pathology: determination and clinical usefulness of the prostate transition zone]. Rev Med Brux. 1998;19:119-23.

20. Deering RE, Bigler SA, King J, Choongkittaworn M, Aramburu E, Brawer MK. Morphometric quantitation of stroma in human benign prostatic hyperplasia. Urology. 1994:44:64-70.

21. Hardman $R L$, Liang $Y$, Ware $S$, et al. Correlation of $P S A$ density to prostate cancer based on prostate volume by 3.0 T MRI. Open J Urol. 2011;1:28-36 
22. Djavan B, Zlotta A, Kratzik C, Remzi M, Seitz C, Schulman CC, et al. PSA, PSA density, PSA density of transition zone, free/total PSA ratio, and PSA velocity for early detection of prostate cancer in men with serum PSA 2.5 to $4.0 \mathrm{ng} / \mathrm{mL}$. Urology. 1999;54:517-22.

23. Taneja SS, Tran K, Lepor H. Volume-specific cutoffs are necessary for reproducible application of prostate-specific antigen density of the transition zone in prostate cancer detection. Urology. 2001;58:222-7.

24. Tanaka N, Fujimoto K, Chihara Y, Torimoto M, Hirao Y, Konishi $\mathrm{N}$, et al. Prostatic volume and volume-adjusted prostatespecific antigen as predictive parameters for prostate cancer patients with intermediate PSA levels. Prostate Cancer Prostatic Dis. 2007;10:274-8.

25. Giannarini G, Scott CA, Moro U, Pertoldi B, Beltrami CA, Selli C. Are PSA density and PSA density of the transition zone more accurate than PSA in predicting the pathological stage of clinically localized prostate cancer? Urol Oncol. 2008;26:353-60.

26. Zlotta AR, Djavan B, Marberger M, Schulman CC. Prostate specific antigen density of the transition zone: a new effective parameter for prostate câncer prediction. J Urol. 1997;157:1315-21.
27. Djavan B, Zlotta AR, Byttebier G, Shariat S, Omar M, Schulman CC, et al. Prostate specific antigen density of the transition zone for early detection of prostate cancer. J Urol. 1998;160:411-8.

28. Lin DW, Gold MH, Ransom S, Ellis WJ, Brawer MK Transition zone prostate specific antigen density: lack of use in prediction of prostatic carcinoma. J Urol. 1998;160:7781-2.

29. Koo KC, Lee DH, Lee SH, Chung BH. Peripheral zone prostate-specific antigen density: an effective parameter for prostate cancer prediction in men receiving $5 \alpha$-reductase inhibitors. Prostate Int. 2013;1:102-8.

Correspondence address: Simon Robinson, MD Department of Urology Frimley Health Foundation Trust Wexham street slough east berkshire SL24HL United Kingdom of Great Britain and Northern Ireland Telephone: +79 81 323-750 E-mail: simon.robinson@fhft.nhs.uk 